KN Kartographische Nachrichten Journal of Cartography and Geographic Information 68. Jahrgang, August 2018, Heft 4 Herausgeber: Deutsche Gesellschaft für Kartographie e.V. (DGfK) - Gesellschaft für Kartographie und Geomatik, vertrete fur Kartographie und Geon
durch den Präsidenten Prof. Dr. Manfred Weisensee

Organ der Deutschen Gesellschaft für Kartographie e.V. (DGfK), der Schweizerischen Gesellschaft für Kartografie (SGK) und der Österreichischen Kartographischen Kommission (OKK) in der Österreichischen Gesellschaft für Geographie (ÖGG).

Schriftleitung

Hauptschriftleiter

Prof. Dr. rer. nat. habil. Mark Vetter (zuständig für Aufsătze, Berichte). Hochschule für angewandte Wissenschaften Würzburg-Schweinfurt, Münzstraße 12, 97070 Würzburg: vetter@kartographische-nachrichten.de

Fon: 0049(0) 931 3511-8249

Weitere Schriftleiter

Prof. Dr. phil. Frank Dickmann (Zweiter Schriftleiter, zuständig für Nachrichten aus Hochschulen und Institutionen, Rezensionen).

Ruhr-Universităt Bochum, Geographisches Institut:

Postfach, 44780 Bochum; frank.dickmann@ @rubde

Fon: 0049(0) 234 32-23379; Fax: 0049(0) 234 32-14964

Dipl.-Ing. Andreas Gollenstede (zuständig für Geoinformation

aktuell) Haareneschstr. 91, 26121 Oldenburg; kn@gollenstedecom

Fon: 0049(0) 441 7779-545; Fax: 0049(0) 441 7779-750

Dr.-Ing. Thomas Chudy (zuständig für DGfK-, SKG- und ÖGG-

Nachrichten). Martin-Luther-Universität Halle-Wittenberg, Institut

für Agrar- und Ernährungswissenschaften, Karl-Freiherr-von-

Fritsch-Straße 4, 06120 Halle/Saale, Fon: 0049(0) 3455522448 :

Fax: 0049(0) 3455527109

Prof. Dr. Reinhard Zölitz (zuständig für Nachrichten aus Hoch-

schulen und Institutionen), Institut für Geographie und Geologie,

Kartographie und GIS, Friedrich-Ludwig-Jahn-Str. 16,

D-17489 Greifswald, Fon 03834864523 , Fax: 03834864501

E-Mail:zoelitz@uni-greifswald.de

Mitarbeit für die Schweiz: Prof. Dr. Lorenz Hurni. Institut für

Kartografie, ETH Hönggerberg, 8093 Zürich; hurni@karto.baug.ethz

ch. Fon:0041 16333033: Fax:00411631153

Mitarbeit für Österreich: Univ.-Prof. Dr. Wolfgang Kainz

Institut für Geographie und Regionalforschung, Universităt Wien,

Universitătsstraße 7/5, 1010 Wien; wolfgang.kainz@univie.ac.at

Fon: 0043 14277-8640; Fax: 0043 14277-9531

Ehrenschriftleiter

Prof. Dr. Jürgen Dodt t, Witten

Dr. rer. nat. h. c. Rolf Harbeck, Bonn

Editorial Board

Prof. Dr. H. Asche, Potsdam; Prof. Dr. Manfred Buchroithner,

Dresden; Prof. Dr. Dirk Burghardt, Dresden; Prof. Dr. D. Dransch.

Potsdam; Prof. Dr. M. Ehlers, Osnabrück, Berlin; Prof. Dr. S. I.

Fabrikant, Zürich, Schweiz; Ass-Prof. Dr. S. Fuhrmann, Fairfax,

USA: Prof. Dr. G. Gartner, Wien, Osterreich; Prof. Dr. D. Grünreich,

Ronnenberg: Dr. Rolf Harbeck Bonn: Prof. Dr. A. Huttermann,

Ronnenberg; Dr. Rolf Harbeck, Bonn, Prof. Dr. A. Hüttermann,

Ludwigsourg; Prof. Dr. L. Hurni, Zurich; Prof. Dr. W. Kainz, Wien;

Prof. Dr. P. Kammerer, München; Prof. Dr. W. G. Koch, Dres
Prof. Dr. Menno-Jan Kraak, Twente, Netherlands; Prof. Dr.

Prof. Dr. Menno-Jan Kraak, Twente, Netherlands; Prof. D
Jukka Krisp, Augsburg; Prof. Dr.-Ing. L. Meng, München;

Prof. Dr. J. Schiewe, Hamburg; PD Dr.-Ing. J. Schoppmeyer, Bonn;

Prof. Dr. J. Schweikart, Berlin; Prof. Dr.-Ing. M. Sester, Hannover:

Prof. Dr. J. Siemer, Regina, Kanada; Prof. Dr. R. Zölitz, Greifswald.

Manuskripte richten Sie bitte an den zuständigen Schriftleiter.

Hinuskise zur Gestaitung des Manustripts finden Sie unter

Hinweise zur Gestalung des Manuskipts finden Sie unter

Reviewverfahren

Wissenschaftliche Beiträge werden im Rahmen eines Peer-ReviewVerfahrens begutachtet. Trotz sorgfältiger Redigierung übernehmen Herausgeber, Redaktion und Verlag keine Haftung für die inhaltliche Richtigkeit der Veröffentlichungen.

Die KN Kartographische Nachrichten ist in der internationalen

Zitationsdatenbank Scopus gelistet

Die DGfK ist Mitglied der

Internationalen Kartographischen

Vereinigung IKV/ICA

Verlag: Kirschbaum Verlag GmbH

Siegfriedstraße 28, D-53179 Bonn

Postfach 210209, D-53157 Bonn

Fon: (0228) 95453-0, Fax: (0228) 95453-27

Internet: http://www.kirschbaum.de, E-Mail: info@kirschbaum.de

Anzeigenleiter: Michael Dietl Nerlagsleiter Fachzeitschriften print

Et digitall. Fon: (0228) 95453-47. E-Mail:m dieti@kirschbaum de:

Anzeigenpreisliste Nr. 53 vom 1.10.2017

Bezugspreise und ISSN: Inland/Ausland Jahresabonnement

inkl. E-Paper und elektronischem Archiv 74,- Euro zzgl.

Versand 9,80,- Euro (jeweils einschl. MwSt.).

Einzelheft 18,- Euro zzgl. Versand

Die Zeitschrift erscheint alle zwei Monate. ISSN 0022-9164. Kündigungsfrist 6 Wochen zum Auslaufen des AbonnementZeitroumes

Satz: EMS Eckert Medienservice, 53881 Euskirchen

Druck: johnen-druck GmbH \& Co. KG, Industriegebiet Bornwiese 54470 Bernkastel-Kues

\title{
Nachwuchssuche auf der INTERGEO
}

\author{
Liebe Leserin, lieber Leser,
}

wer gegenwärtig ein Studium oder eine Ausbildung im Bereich der Geomatik aufgenommen hat, kann sich glücklich schätzen. Eine gut bezahlte Anstellung ist ziemlich wahrscheinlich. Natürlich muss man sich anstrengen, als Azubi oder als Studierender - dies gilt für alle Berufsausbildungen oder Studienfächer. Ferner sollte man sich ein Steckenpferd aussuchen, ein Thema, das besonders interessiert: z. B. Programmierung und Anwendung von GIS,

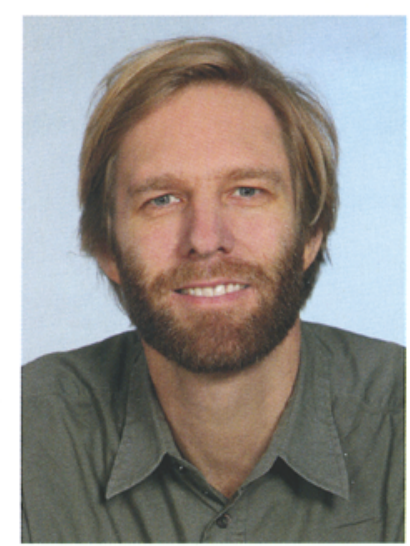

Mark Vetter präzise Industrievermessung mit Laserscannern, drohnengestützte Geländeaufnahmen, autonomes Fahren über exakte Geodaten, Auswertung von Fernerkundungsdaten von den Sentinel-Satelliten - alles spannende Themenfelder. Hier wird sich viel tun in den nächsten Jahren, hier werden Spezialistinnen und Spezialisten gesucht. Die Branche braucht Berufsnachwuchs, der sich mit diesen Technologien und den entsprechenden Inhalten auskennt.

Die Verantwortlichen für die INTERGEO 2018, die dieses Jahr in den Messehallen von Frankfurt am Main stattfındet, haben dies erkannt. Daher ist die Förderung der Nachwuchsgewinnung für die Branche eines der zentralen Leitmotive der diesjährigen Fachmesse. Auszubildende und Studierende sollten dies nutzen: Angesprochen sind also die jungen Geomatikerinnen und Geomatiker in Ausbildung oder Studium: Gehen Sie auf die INTERGEO, sprechen Sie die verschiedenen Aussteller an, erzählen Sie über Ihre Interessen oder Studienschwerpunkte. Fragen Sie nach Praktika oder ob Sie eine E-Mail mit Ihren Bewerbungsunterlagen schicken dürfen. Sie werden sehen, in den wenigsten Fällen werden Sie eine ablehnende Haltung erfahren. Ganz im Gegenteil, Sie werden das Gefühl haben, Sie werden gebraucht. Und Sie können sich das Betätigungsfeld bzw. die Firma aussuchen, die am besten Ihren Vorstellungen entspricht.

Im vorliegenden INTERGEO-Heft können Sie folgende Artikel lesen: Lilja Friesen zeigt federführend auf, wie situationsbezogen Gesundheitsdaten in interaktiven Webmapping-Applikationen bereitgestellt werden können. Das Team um Thomas Kersten stellt am Beispiel einer historischen Rekonstruktion von Bad Segeberg die Möglichkeiten der virtuellen Realität für interaktive 3D-Darstellungen dar. Stefan Neumeier hat sich einem klassischen GIS-Thema anwendungsbezogen genähert: der Erreichbarkeit von Einsatzzielen der Polizei an verschiedenen Orten in Deutschland. Und letztlich sorgt Francis Harvey dafür, dass der World Geo-Graphic Atlas von Herbert Bayer aus dem Jahr 1953 nicht in Vergessenheit gerät und fragt, welche Bedeutung er heute hat.

Viel Freude beim Lesen dieser KN-Ausgabe und eine inspirierende INTERGEO wünscht Ihnen ihr

\section{Morunter}

